\title{
IFITM1, CD10, SMA, and h-caldesmon as a helpful combination in differential diagnosis between endometrial stromal sarcoma and cellular leiomyoma
}

\section{Weilin Zhao}

Department of Pathology, The First Affiliated Hospital, Shihezi University School of Medicine, Xinjiang 832002 , China

\section{Cui Mei}

Department of Pathology, Xinjiang Uygur Autonomous Region People's Hospital, Xinjiang 832002, China Xin Hua Ji

Department of Pathology, The First Affiliated Hospital, Shihezi University School of Medicine, Xinjiang 832002 , China

\section{Xin Xiong}

Department of Pathology, The First Affiliated Hospital, Shihezi University School of Medicine, Xinjiang 832002 , China

\section{Xi Hua Shen}

Department of Pathology, The First Affiliated Hospital, Shihezi University School of Medicine, Xinjiang 832002 , China

\section{Lin Tao}

Department of Pathology, The First Affiliated Hospital, Shihezi University School of Medicine, Xinjiang 832002 , China

\section{Wei Jia}

Department of Pathology, The First Affiliated Hospital, Shihezi University School of Medicine, Xinjiang 832002, China

\section{Li Juan Pang}

Department of Pathology, The First Affiliated Hospital, Shihezi University School of Medicine, Xinjiang 832002 , China

\section{Zhen Zhu Sun}

Department of Pathology, Xinjiang Uygur Autonomous Region People's Hospital, Xinjiang 832002, China

\section{Chun Wang}

Department of Pathology, Xinjiang Uygur Autonomous Region People's Hospital, Xinjiang 832002, China

\section{Hong Zou ( $\square$ zouhong_patho@163.com )}

Department of Pathology, Shihezi University School of Medicine, Xinjiang 832002, China https://orcid.org/0000-0003-3051-8006 


\section{Research}

Keywords: IFITM1, CD10, SMA, h-caldesmon, Immunohistochemical, Differential Diagnosis, ESS,CL Posted Date: February 11th, 2020

DOl: https://doi.org/10.21203/rs.2.23242/v1

License: (1) (1) This work is licensed under a Creative Commons Attribution 4.0 International License. Read Full License 


\section{Abstract}

The differential diagnosis of endometrial stromal sarcoma (ESS) and uterine cellular leiomyoma (CL) remains a challenge in clinical practice. Cluster of differentiation 10 (CD10) and smooth muscle actin (SMA) are commonly used in the differential diagnosis of ESS and CL. However, the current combination of immunohistochemical antibodies has been shown to be inaccurate, suggesting the need for novel immunomarkers panels for differentiating between ESS and CL. Interferon-induced transmembrane protein 1 (IFITM1) is a novel immunomarker for endometrial stromal cells, h-caldesmon is an immunomarker for smooth muscle cells and has a higher specificity than SMA. So this study aimed to investigate IFITM1, CD10, SMA, and h-caldesmon as a useful combination of biomarkers for diagnosing between ESS and CL. Tissue microarrays were used to detect IFITM1, CD10, SMA, and h-caldesmon immunohistochemical staining in $30 \mathrm{ESS}$ and $33 \mathrm{CL}$ cases. The expressions of IFITM1 and CD10 were high in ESS (86.7\% and 63.3\%, respectively) but low in CL (18.2\% and $21.2 \%)$, whereas those of $h$ caldesmon and SMA were high in both CL (87.9\% and 100\%) and low in ESS (6.9\% and $40 \%)$. In diagnosing ESS, IFITM1 had better sensitivity and specificity ( $86.7 \%$ and $81.8 \%$, respectively) than CD10 (63.3\% and $78.8 \%)$. The specificity of h-caldesmon in diagnosing CL was significantly higher $(93.1 \%)$ than that of SMA $(60 \%)$. When all four antibodies were combined for the differential diagnosis, the area-underthe-curve predictive value was 0.995 . The most sensitive and specific combinations for diagnosing ESS were IFITM1(+) or CD10(+) and h-caldesmon(-) ( sensitivity $86.7 \%$, specificity $93.9 \%$ ), IFITM1(+) and hcaldesmon(-) ((sensitivity $80 \%$, specificity $100 \%)$. The most sensitive and specific combinations for diagnosing $\mathrm{CL}$ were h-caldesmon(+) and SMA(+)(sensitivity $87.9 \%$, specificity $100 \%)$, h-caldesmon(+) or SMA(+) and IFITM1(-)(sensitivity $81.8 \%$, specificity 93.1\%). Therefore, IFITM1, CD10, SMA, and hcaldesmon are a good combination of biomarkers for the differential diagnosis of ESS and CL.The differential diagnosis of endometrial stromal sarcoma (ESS) and uterine cellular leiomyoma (CL) remains a challenge in clinical practice. Cluster of differentiation 10 (CD10) and smooth muscle actin (SMA) are commonly used in the differential diagnosis of ESS and CL. However, the current combination of immunohistochemical antibodies has been shown to be inaccurate, suggesting the need for novel immunomarkers panels for differentiating between ESS and CL. Interferon-induced transmembrane protein 1 (IFITM1) is a novel immunomarker for endometrial stromal cells, h-caldesmon is an immunomarker for smooth muscle cells and has a higher specificity than SMA. So this study aimed to investigate IFITM1, CD10, SMA, and h-caldesmon as a useful combination of biomarkers for diagnosing between ESS and CL. Tissue microarrays were used to detect IFITM1, CD10, SMA, and h-caldesmon immunohistochemical staining in $30 \mathrm{ESS}$ and $33 \mathrm{CL}$ cases. The expressions of IFITM1 and CD10 were high in ESS (86.7\% and 63.3\%, respectively) but low in CL (18.2\% and $21.2 \%)$, whereas those of $h$ caldesmon and SMA were high in both CL (87.9\% and 100\%) and low in ESS (6.9\% and $40 \%)$. In diagnosing ESS, IFITM1 had better sensitivity and specificity ( $86.7 \%$ and $81.8 \%$, respectively) than CD10 (63.3\% and 78.8\%). The specificity of h-caldesmon in diagnosing CL was significantly higher (93.1\%) than that of SMA $(60 \%)$. When all four antibodies were combined for the differential diagnosis, the area-underthe-curve predictive value was 0.995 . The most sensitive and specific combinations for diagnosing ESS were IFITM1(+) or CD10(+) and h-caldesmon(-) ( sensitivity $86.7 \%$, specificity $93.9 \%$ ), IFITM1(+) and h- 
caldesmon(-) ((sensitivity $80 \%$, specificity $100 \%)$. The most sensitive and specific combinations for diagnosing $\mathrm{CL}$ were h-caldesmon(+) and SMA(+)(sensitivity $87.9 \%$, specificity $100 \%$ ), h-caldesmon(+) or SMA(+) and IFITM1(-)(sensitivity $81.8 \%$, specificity 93.1\%). Therefore, IFITM1, CD10, SMA, and hcaldesmon are a good combination of biomarkers for the differential diagnosis of ESS and CL.The differential diagnosis of endometrial stromal sarcoma (ESS) and uterine cellular leiomyoma (CL) remains a challenge in clinical practice. Cluster of differentiation 10 (CD10) and smooth muscle actin (SMA) are commonly used in the differential diagnosis of ESS and CL. However, the current combination of immunohistochemical antibodies has been shown to be inaccurate, suggesting the need for novel immunomarkers panels for differentiating between ESS and CL. Interferon-induced transmembrane protein 1 (IFITM1) is a novel immunomarker for endometrial stromal cells, h-caldesmon is an immunomarker for smooth muscle cells and has a higher specificity than SMA. So this study aimed to investigate IFITM1, CD10, SMA, and h-caldesmon as a useful combination of biomarkers for diagnosing between ESS and CL. Tissue microarrays were used to detect IFITM1, CD10, SMA, and h-caldesmon immunohistochemical staining in 30 ESS and $33 \mathrm{CL}$ cases. The expressions of IFITM1 and CD10 were high in ESS (86.7\% and 63.3\%, respectively) but low in CL (18.2\% and $21.2 \%)$, whereas those of $h$ caldesmon and SMA were high in both CL (87.9\% and 100\%) and low in ESS (6.9\% and $40 \%)$. In diagnosing ESS, IFITM1 had better sensitivity and specificity ( $86.7 \%$ and $81.8 \%$, respectively) than CD 10 (63.3\% and $78.8 \%)$. The specificity of h-caldesmon in diagnosing CL was significantly higher $(93.1 \%)$ than that of SMA $(60 \%)$. When all four antibodies were combined for the differential diagnosis, the area-underthe-curve predictive value was 0.995 . The most sensitive and specific combinations for diagnosing ESS were IFITM1(+) or CD10(+) and h-caldesmon(-) ( sensitivity $86.7 \%$, specificity $93.9 \%$ ), IFITM1(+) and hcaldesmon(-) ((sensitivity $80 \%$, specificity $100 \%)$. The most sensitive and specific combinations for diagnosing $\mathrm{CL}$ were h-caldesmon(+) and SMA(+)(sensitivity $87.9 \%$, specificity $100 \%$ ), h-caldesmon(+) or SMA(+) and IFITM1(-)(sensitivity $81.8 \%$, specificity 93.1\%).Therefore, IFITM1, CD10, SMA, and hcaldesmon are a good combination of biomarkers for the differential diagnosis of ESS and CL.

\section{Background}

Endometrial stromal sarcoma (ESS) is a rare malignant mesenchymal tumor of the uterine. In 2014, the World Health Organization classified ESS as low-grade ESS, high-grade ESS, and undifferentiated endometrial sarcoma[1]. However, there is a overlap in morphology and immunohistochemistry between ESS and leiomyoma, especially for the low grade ESS from cellular leiomyoma (CL). Currently, cluster of differentiation 10 (CD10) has been considered as the best immunomarker for endometrial stromal cells[2-6], but it not expressed in all mesenchymal tumors[7-9]. Rather, CD10 is sometimes expressed in leiomyoma[10,11]. Smooth muscle actin (SMA) is a common biomarker for smooth muscle, however, SMA is sometimes expressed in ESS [12-15], suggesting the need for novel immunomarkers and immunohistochemical panels for differentiating between ESS and CL.

Interferon-induced transmembrane protein 1 (IFITM1), also called CD225, is a novel immunomarker for endometrial stromal cells and tumors[16, 17] and outperforms CD10 in distinguishing LG ESS from CL[18, 19]. Meanwhile, h-caldesmon is an immunomarker for smooth muscle cells and has a higher specificity 
than smooth muscle actin (SMA). However, there has been no study on the combined use of IFITM1, CD10, SMA, and h-caldesmon in distinguishing between ESS and CL. This study aimed to investigate IFITM1, CD10, SMA, and h-caldesmon as a useful combination of biomarkers for the differential diagnosis of ESS and CL.

\section{Materials And Methods}

The study were approved by the Review Boards of First Affiliated Hospital, Shihezi University School of Medicine and Xinjiang Uygur Autonomous Region People's Hospital.

\section{Clinical data}

This study enrolled 30 patients with ESS ( 5 with endometrial stromal nodules, 16 with LG ESS, 5 with highgrade ESS, and 4 with undifferentiated endometrial sarcoma) and 33 patients with CL. Data were collected from 2012 to 2017 from the Department of Pathology of the First Affiliated Hospital of Shihezi University School of Medicine and the Department of Pathology of Xinjiang Uygur Autonomous Region People's Hospital. The average age of 30 ESS patients was 49.8 (27-73) years, and the main clinical symptoms were irregular vaginal bleeding, abdominal pain, postmenopausal vaginal bleeding, and uterine fibroids. The $33 \mathrm{CL}$ patients had an average age of $40.2(26-60)$ years and mainly showed clinical manifestations of dysmenorrhea, prolonged menstrual period, and increased menstrual volume. All pertinent clinical information was obtained from the hospital electronic medical records. All patients had complete medical history and clinicopathologic data, and all cases were confirmed by surgery and pathology.

\section{Tissue microarray building}

Paraffin blocks and corresponding hematoxylin and eosin (HE)-stained sections were collected, and the HE-stained sections were evaluated by two senior pathologists. Morphologically representative regions were carefully selected on each individual paraffin-embedded block, and a hollow needle $(1.0 \mathrm{~mm}$ diameter) was used to puncture the selected area to a new small wax block. Considering the specificity of the tumor and the tendency of the paraffin tissue to flake off, two punctures were performed in different areas of each tumor wax block.

\section{Immunohistochemistry}

For immunohistochemical analysis, biopsy specimens were fixed in $10 \%$ neutral-buffered formalin and routinely processed. The paraffin-embedded blocks were sectioned ( $4 \mu \mathrm{m}$ thickness), stained with $\mathrm{HE}$, and observed by microscopy. The two-step immunohistochemical EnVision method was applied. The primary antibodies used are listed in Table 1 . The extent of staining was evaluated as $0 \%, 0-25 \%, 26-50 \%, 51-$ $75 \%$, and $76-100 \%$, and the intensity of staining as absent (0), weak (1+), moderate (2+), and strong (3+). The second staining was conducted after 1 month, and the results were interpreted as described above. When a different staining evaluation was used, the higher intensity score was used as the final score. 
Table 1

Commercial Sources and Characteristics of IFITM1,CD10,h-caldesmon and SMA Antibodies.

\begin{tabular}{|lllll|}
\hline Name & Dilution & Company & Antigen retrieval & Location \\
\hline IFITM1 & $1: 400$ & Sigma & citrate & Nucleus/plasma \\
\hline CD10 & $1: 50$ & ZSGB-BIO & EDTA & Cytoplasm/membrane \\
SMA & $1: 100$ & ZSGB-BIO & citrate & Cytoplasm \\
\hline h-caldesmon & $1: 100$ & ZSGB-BIO & citrate & Cytoplasm \\
\hline
\end{tabular}

\section{Statistical analysis}

Differences between the two groups were compared using the chi-squared test. The sensitivity, specificity, and positive predictive values (PPVs) were calculated from the screening and diagnostic tests. The staining score was obtained by multiplying the extent with the intensity, and the resulting score was used for the receiver operating characteristic curve. All statistical analyses were performed using SPSS version 17.0. A p-value of $<0.05$ was considered as statistically significant.

\section{Results}

The immunohistochemical results are summarized in Table 2 and illustrated in Fig. 1. The sensitivity, specificity, PPVs, and negative predictive values (NPVs) are summarized in Tables 3-6 and shown in Figs. 2-4.

Table 2

Intensity and Extent of Immunohistochemical Staining of IFITM1,CD10,h-caldesmon and SMA in endometrial stromal sarcoma and celluar leiomyoma.

\begin{tabular}{|c|c|c|c|c|c|c|c|c|c|c|}
\hline \multirow[t]{3}{*}{ Antibodies } & \multicolumn{5}{|c|}{ ESS(30 cases) } & \multicolumn{5}{|c|}{ CL(33 cases) } \\
\hline & \multirow[t]{2}{*}{ Positive } & \multicolumn{4}{|c|}{ Category Intensity } & \multirow[t]{2}{*}{ Positive } & \multicolumn{4}{|c|}{ Category Intensity } \\
\hline & & 0 & $1+$ & $2+$ & $3+$ & & 0 & $1+$ & $2+$ & $3+$ \\
\hline IFITM1 & $26(86.7 \%)$ & 4 & 10 & 8 & 8 & $6(18.2 \%)$ & 27 & 6 & 0 & 0 \\
\hline CD10 & $19(63.3 \%)$ & 11 & 6 & 7 & 6 & $7(21.2 \%)$ & 26 & 7 & 0 & 0 \\
\hline $\begin{array}{l}\text { h- } \\
\text { caldesmon }\end{array}$ & $2(6.7 \%)$ & 28 & 2 & 0 & 0 & $29(87.9 \%)$ & 4 & 15 & 10 & 4 \\
\hline SMA & $12(40 \%)$ & 18 & 4 & 6 & 2 & $33(100 \%)$ & 0 & 22 & 9 & 2 \\
\hline
\end{tabular}


Table 3

Sensitivity, Specificity, Positive Predictive Value and Negative Predictive Value of IFITM1 and CD10 for endometrial stromal sarcoma.

\begin{tabular}{|ccccc|}
\hline Antibodies & Sensitivity (\%) & Specificity (\%) & PPV(\%) & NPV(\%) \\
\hline IFITM1 & 86.7 & 81.8 & 81.3 & 87.1 \\
\hline CD10 & 63.3 & 78.8 & 73.1 & 70.3 \\
\hline \multicolumn{2}{|l}{ Abbreviations: PPV indicates Positive Predictive Value, NPV indicates Negative Predictive Value. } \\
\hline
\end{tabular}

\section{IFITM1 and CD10}

Both ESS (Fig. 1-A) and CL cases (Fig. 1-B) showed a dense spindle-cell braid-like arrangement. Among the 30 ESS cases, 26 (86.7\%) demonstrated IFITM1 nuclear positivity (Fig. 1-C). The staining intensity was strong (3+) in 8 cases, moderate (2+) in 8 cases, and weak (1+) in 10 cases. The average intensity score was 1.7. Of the $33 \mathrm{CL}$ cases, only 6 (18.2\%) demonstrated IFITM1 nuclear positivity (Fig. 1-D), all of which scored weak (1+) in intensity. CD10 was expressed in 19 (63.3\%) of the 30 ESS cases (Fig. 1-E). The staining in these cases occurred in the cell cytoplasm and was strong (3+) in 6 cases, moderate (2+) in 7 cases, and weak (1+) in 6 cases. The average intensity score was 1.3. Only $7(21.2 \%)$ of the $33 \mathrm{CL}$ cases were CD10(+), and all positive cases had a weak (1+) intensity (Fig. 1-F).

\section{SMA and h-caldesmon}

SMA was positive in $12(40 \%)$ of the 30 ESS cases (Fig. 1-G). The staining in these cases was expressed in the cytoplasm and was moderate to strong $(2+$ to $3+)$ in 8 cases and weak $(1+)$ in 4 cases. All 33 (100\%) CL cases expressed SMA (Fig. 1-H), and among them, the staining was moderate to strong ( $2+$ to $3+)$ in 11 cases and weak (1+) in the remaining cases. The average intensity score was 1.7 . Meanwhile, hcaldesmon was expressed in the cell cytoplasm of only 2 (6.7\%) of the 30 ESS cases (Fig. 1-I), and the staining in these positive cases were weak (1+). However, $29(87.9 \%)$ of the $33 \mathrm{CL}$ cases exhibited hcaldesmon positivity (Fig. 1-J). In these $33 \mathrm{CL}$ cases, the staining was strong (3+) in 4 cases, moderate $(2+)$ in 10 cases, and weak (1+) in 15 cases. The average intensity score was 1.4 .

\section{Sensitivity, specificity, PPVs, and NPVs of IFITM1, CD10, h- caldesmon, and SMA}

In the diagnosis of ESS, IFITM1 showed a sensitivity of $86.7 \%$, a specificity of $81.8 \%$, a PPV of $81.3 \%$, and an NPV of $87.1 \%$. For CD10, the sensitivity, specificity, PPV, and NPV were $63.3 \%, 78.8 \%, 73.1 \%$, and $70.3 \%$, respectively. h-caldesmon positivity may support a diagnosis of $\mathrm{CL}$, showing a sensitivity of $87.9 \%$, a specificity of $93.3 \%$, a PPV of $93.5 \%$, and an NPV of $87.5 \%$. SMA had the highest sensitivity (100\%), but its specificity was $60 \%$, significantly lower than that of h-caldesmon. SMA had a PPV and an NPV of $73.3 \%$ and $100 \%$, respectively (Tables 3 and 4 ). 
Table 4

Sensitivity, Specificity, Positive Predictive Value and Negative Predictive Value of h-caldesmon and SMA for celluar leiomyoma.

\begin{tabular}{|cllll|}
\hline Antibodies & Sensitivity (\%) & Specificity (\%) & PPV(\%) & NPV(\%) \\
\hline h-caldesmon & 87.9 & 93.3 & 93.5 & 87.5 \\
\hline SMA & 100.0 & 60.0 & 73.3 & 100.0 \\
\hline Abbreviations: PPV indicates Positive Predictive Value, NPV indicates Negative Predictive Value. \\
\hline
\end{tabular}

\section{IFITM1, CD10, h-caldesmon, and SMA as a useful combination for differential diagnosis}

Based on the expressions of the four antibodies and their receiver operating characteristic curve(the areaunder-the-curve predictive value was 0.995 (Fig. 2), we speculate that their combinations could be helpful in the differential diagnosis of ESS and CL.

When all four antibodies were combined for the ESS diagnosis (Table 5, Fig. 3), The most sensitive combination was IFITM1(+) or CD10(+), IFITM1(+) or CD10(+) and h-caldesmon(-), IFITM1(+) and hcaldesmon(-), with a sensitivity of $93.3 \%, 86.7 \%, 80 \%$, respectively (Fig. 3-A). The combination of antibodies greatly increased the specificity of ESS diagnosis (Fig. 3-B), the specificity of combinations of IFITM1(+) and h-caldesmon(-), IFITM1(+) and SMA(-), IFITM1(+) and CD10(+) and h-caldesmon(-), IFITM1(+) and CD10(+) and h-caldesmon(-) and SMA(-), and IFITM1(+) or CD10(+) and h-caldesmon(-) and SMA $(-)$ were $100 \%$. Considering both sensitivity and specificity, the combination with the best diagnostic value for ESS was IFITM1(+) or CD10(+) and h-caldesmon(-), with a sensitivity and a specificity of $86.7 \%$ and $93.9 \%$, respectively. 
Table 5

The Sensitivity and Specificity of combined IFITM1, CD10, h-caldesmon and SMA immunostaining in the Diagnosis of endometrial stromal sarcoma.

\begin{tabular}{|lll|}
\hline Groups & Sensitivity (\%) & Specificity (\%) \\
\hline IFITM1 (+) and h-caldesmon(-) for ESS & 80.0 & 100.0 \\
\hline IFITM1 (+) and SMA(-) for ESS & 60.0 & 97.0 \\
\hline CD10(+) and h-caldesmon(-) for ESS & 50.0 & 100.0 \\
\hline CD10(+) and SMA(-) for ESS & 36.7 & 100.0 \\
\hline IFITM1 (+) and CD10(+) for ESS & 56.7 & 93.9 \\
\hline IFITM1 (+) or CD10(+) for ESS & 93.3 & 66.7 \\
\hline IFITM1 (+) or CD10(+) and h-caldesmon(-) for ESS & 86.7 & 93.9 \\
\hline IFITM1 (+) and CD10(+) and h-caldesmon(-) for ESS & 53.3 & 100.0 \\
\hline IFITM1 (+) or CD10(+) and SMA(-) for ESS & 56.7 & 100.0 \\
\hline IFITM1 (+) and CD10(+) and SMA(-) for ESS & 30.0 & 100.0 \\
\hline IFITM1 (+) and CD10(+) and h-caldesmon(-) and SMA(-) for ESS & 31.0 & 100.0 \\
\hline IFITM1 (+) or CD10(+) and h-caldesmon(-) and SMA(-) for ESS & 56.7 & 100.0 \\
\hline Abbreviations: ESS indicates Endometrial Stromal Sarcomas. & & \\
\hline
\end{tabular}

In diagnosing $\mathrm{CL}$ (Table 6, Fig. 4), the combinations h-caldesmon(+) or SMA(+), h-caldesmon(+) and SMA (+), and h-caldesmon(+) or SMA(+) and IFITM1(-) showed better sensitivity for differentiating CL from ESS, with sensitivity values of $100 \%, 87.9 \%$, and $81.8 \%$, respectively(Fig. 4-A). On the other hand, hcaldesmon(+) and IFITM1(-), h-caldesmon(+) and SMA(+), h-caldesmon(+) and SMA(+) and IFITM1(-), and h-caldesmon(+) and SMA(+) and IFITM1(-) and CD10(-) showed better specificity for predicting $\mathrm{CL}$ from ESS, with all specificity were $100 \%$ (Fig. 4-B). Taking into account both sensitivity and specificity, hcaldesmon(+) and SMA(+) was the best combination for distinguishing CL from ESS, with a sensitivity of $87.9 \%$ and a specificity of $100 \%$. The second best combination for distinguishing CL from ESS was $h-$ caldesmon(+) or SMA(+) and IFITM1(-), with a sensitivity of $81.8 \%$ and a specificity of $93.1 \%$. 
Table 6

The Sensitivity and Specificity of combined IFITM1,CD10,h-caldesmon and SMA immunostaining in the Diagnosis of celluar leiomyoma.

\begin{tabular}{|lll|}
\hline Groups & Sensitivity (\%) & Specificity (\%) \\
\hline h-caldesmon(+) andIFITM1(-) for CL & 69.7 & 100.0 \\
\hline SMA(+) and IFITM1(-) for CL & 81.8 & 93.1 \\
\hline h-caldesmon(+) and CD10(-) for CL & 72.7 & 96.6 \\
\hline SMA(+) and CD10(-) for CL & 78.8 & 82.8 \\
\hline h-caldesmon(+) and SMA(+) for CL & 87.9 & 100.0 \\
\hline h-caldesmon(+) or SMA(+) for CL & 100.0 & 57.1 \\
\hline h-caldesmon(+) and SMA(+) and IFITM1(-) for CL & 69.7 & 100.0 \\
\hline h-caldesmon(+) or SMA(+) and IFITM1(-) for CL & 81.8 & 93.1 \\
\hline h-caldesmon(+) and SMA(+) and CD10(-) for CL & 72.7 & 96.6 \\
\hline h-caldesmon(+) or SMA(+) and CD1(-) for CL & 78.8 & 82.8 \\
\hline h-caldesmon(+) and SMA(+) and IFITM1(-) and CD10(-) for CL & 57.6 & 100.0 \\
\hline h-caldesmon(+) or SMA(+) and IFITM1(-) and CD10(-) for CL & 66.7 & 93.1 \\
\hline Abbreviations: CL indicates celluar leiomyoma. & & \\
\hline
\end{tabular}

\section{Discussion}

The standard routine immunomarker panel used by most pathologists to distinguish ESS from CL consists of CD10, h-caldesmon, and SMA [10, 20-22], and an immunoprofile of CD10(+), h-caldesmon(-), and SMA(-) supports the diagnosis of ESS [15]. However, the current combination of immunohistochemical antibodies has been shown to be inaccurate, especially when diagnosing endometrial stromal tumors using CD10 alone $[3,10]$. CD10 is not merely expressed in endometrial stromal tumors but is also positively expressed in $20-30 \%$ of smooth muscle tumors $[13,15]$. SMA is a common muscle marker for endometrial stromal tumors and therefore has a very low specificity. Although h-caldesmon has a higher specificity that of SMA, its sensitivity is worse[10, 13, 15, 23]. Thus, the need for a novel biomarker or a new immunohistochemical combination is imperative.

IFITM1 is a novel biomarker for endometrium stromal cells and is reported to be more valuable than CD10 $[19,24]$. According to Busca et al[19], IFITM1 and CD10 were expressed in 14 ESS cases, and although their sensitivities were $83 \%$ and $91 \%$, respectively, IFITM1 showed a higher specificity than CD 10 , that is, $70 \%$ vs. $45 \%$. These findings are consistent with our findings, which state that IFITM1 was more specific and sensitive than CD10 in endometrial stromal tumors (sensitivity $86.7 \%$ vs. $63.3 \%$, specificity $81.8 \%$ vs. 
78.8\%). Rush et al.[13] compared the expressions of SMA and h-caldesmon between ESS and CL and found that SMA was more sensitive than h-caldesmon ( $90.9 \%$ vs. $72.7 \%$ ); however, h-caldesmon was more specific than SMA (100\% vs. $91.7 \%)$. In our study, h-caldesmon showed a lower sensitivity than SMA ( $87.9 \%$ vs. $100 \%)$, but its specificity was significantly higher ( $93.3 \%$ vs. $60 \%)$.

Based on the expressions of the four antibodies and their receiver operating characteristic curve, we speculate that their combination could be useful in the clinical and differential diagnosis of ESS and CL. When such combination was used for diagnosis, the area-under-the-curve predictive value was 0.995 . However, there are certain limitations in that the receiver operating characteristic curve cannot completely show the positive and negative expressions of the antibodies. We therefore compared the sensitivities and specificities of the four combined antibodies using a screening test and selected the best biomarker panel. For the diagnosis of ESS, the combination IFITM1(+) or CD10(+) had the highest sensitivity (93.3\%), followed by IFITM1(+) or CD10(+) and h-caldesmon(-) (86.7\%). In terms of specificity, the combinations IFITM1(+) and h-caldesmon(-), IFITM1(+) and SMA(-), and CD10(+) and SMA(-) were the best panels, having a specificity of up to $100 \%$, while IFITM1(+) or CD10(+) was the worst panel, with a specificity of only $66.7 \%$. Considering both sensitivity and specificity, the best panel for diagnosing ESS was IFITM1(+) or CD10(+) and h-caldesmon(-), with a sensitivity of $86.7 \%$ and a specificity of $93.9 \%$. The other good predictive panel for distinguishing ESS from CL was IFITM1(+) and h-caldesmon(-), with a sensitivity of $80 \%$ and a specificity of $100 \%$.

In general, no one immunomarker is sensitive and specific enough to make an accurate diagnosis, and so we usually use an immunohistochemical panel for differential diagnosis. This study revealed that the combination of IFITM1, CD10, SMA, and h-caldesmon comprised the best immunohistochemical panel for differentiating between ESS and CL. Considering the costs, we also recommend the combinations IFITM1 and h-caldesmon for the same purpose.

\section{Conclusion}

Immunohistochemical combinations of the novel antibody IFITM1 with traditional antibodies CD10, SMA, and h-caldesmon can be very useful in distinguishing ESS from CL, especially when the clinical history and histological morphology cannot be differentiated totally.

\section{Declarations}

\section{Acknowledgments}

This work were supported by the National Natural Science Foundation of China [grant numbers 81460383, 81660411], the International Cooperation Project of Xinjiang Production and Construction Corps of China [grant number 2019BC001], the Key Areas Innovation Team Project of Xinjiang Production and Construction Corps of China [grant number 2018CB002]. 
Ethical approval was obtained from the Institutional Ethics Review Board (IERB) of the First Affiliated Hospital of School of Medicine, Shihezi University.Research was conducted according to all ethical standards, and written informed consent was obtained from all patients.

\section{Consent for publication}

Consent to publish has been obtained from all authors.

\section{Availability of data and materials}

All data in our study are available upon request.

\section{Competing interests}

The authors have no conflicts of interest to declare. No funding or other benefits related to the subject of this article were received from any commercial entity.

\section{Fundings}

This work were supported by the National Natural Science Foundation of China [grant numbers 81460383, 81660411], the International Cooperation Project of Xinjiang Production and Construction Corps of China [grant number 2019BC001], the Key Areas Innovation Team Project of Xinjiang Production and Construction Corps of China [grant number 2018CB002].

\section{Authors' contributions}

ZH , SZZ and WC conceived and designed this study; CM provided the clinical specimens; ZWL, JXH and XX performed the experiments; SXH ,JW and TL acquired the data; ZWL and CM analyzed the data and results; ZWL wrote the manuscript; $Z \mathrm{H}$ and PLJ improved and revised the manuscript. All authors read and approved the final manuscript.

\section{References}

1. Conklin, C.M. and T.A. Longacre, Endometrial stromal tumors: the new WHO classification. 2014. 21(6): p. 383.

2. Toki, T., et al., CD10 is a Marker for Normal and Neoplastic Endometrial Stromal Cells. International Journal of Gynecological Pathology Official Journal of the International Society of Gynecological Pathologists. 21(1): p. 41-47.

3. Chu, P.G., et al., Utility of CD10 in Distinguishing between Endometrial Stromal Sarcoma and Uterine Smooth Muscle Tumors: An Immunohistochemical Comparison of 34 Cases. Mod Pathol. 14(5): p. 465-471.

4. MCCLUGGAGE, W., CD10 is a sensitive and diagnostically useful immunohistochemical marker of normal endometrial stroma and endometrial stromal neoplasms. Histopathology, 2001. 39. 
5. Vera, A.A. and M.B. Guadarrama, Endometrial stromal sarcoma: clinicopathological and immunophenotype study of 18 cases. 2011. 15(5): p. 312-317.

6. Oliva and Esther, CD10 Expression in the Female Genital Tract. Advances in Anatomic Pathology. 11(6): p. 310-315.

7. Groisman, G.M. and A. Meir, CD10 is helpful in detecting occult or inconspicuous endometrial stromal cells in cases of presumptive endometriosis. Archives of Pathology \& Laboratory Medicine, 2003. 127(8): p. 1003-1006.

8. Potlog-Nahari, C., et al., CD10 immunohistochemical staining enhances the histological detection of endometriosis. Fertility \& Sterility. 82(1): p. 86-92.

9. Sumathi, V., CD10 is useful in demonstrating endometrial stroma at actopic sites and in confirming a diagnosis of endometrosis. Journal of Clinical Pathology, 2002. 55.

10. Abeler, V.M. and M. Nenodovic, Diagnostic Immunohistochemistry in Uterine Sarcomas: A Study of 397 Cases. International Journal of Gynecological Pathology Official Journal of the International Society of Gynecological Pathologists, 2011. 30(3): p. 236-243.

11. Comparative clinicopathologic and immunohistochemical analysis of uterine sarcomas diagnosed using the World Health Organization classification system. 40(11): p. 0-1585.

12. Oliva, E., et al., An Immunohistochemical Analysis of Endometrial Stromal and Smooth Muscle Tumors of the gUterus. American Journal of Surgical Pathology. 26(4): p. 403-412.

13. Rush, D.S., et al., h-caldesmon, a novel smooth muscle-specific antibody, distinguishes between cellular leiomyoma and endometrial stromal sarcoma. Am J Surg Pathol, 2001. 25(2): p. 253-8.

14. Zhu, X.Q., et al., Immunohistochemical markers in differential diagnosis of endometrial stromal sarcoma and cellular leiomyoma. Gynecologic Oncology. 92(1): p. 71-79.

15. Mittal, K., R. Soslow, and W.G. Mccluggage, Application of Immunohistochemistry to Gynecologic Pathology. Archives of Pathology \& Laboratory Medicine, 2008. 132(3): p. 402-423.

16. Sun, H., et al., IFITM1 is a Novel, Highly Sensitive Marker for Endometriotic Stromal Cells in Ovarian and Extragenital Endometriosis. Reproductive Sciences.

17. Parra-Herran, C.E., et al., Targeted development of specific biomarkers of endometrial stromal cell differentiation using bioinformatics: the IFITM1 model. Modern Pathology An Official Journal of the United States \& Canadian Academy of Pathology Inc. 27(4): p. 569-579.

18. Park, H.J., et al., Characterisation of mouse interferon-induced transmembrane protein-1 gene expression in the mouse uterus during the oestrous cycle and pregnancy. Reprod Fertil Dev, 2011. 23.

19. Busca, A., et al., IFITM1 Outperforms CD10 in Differentiating Low-grade Endometrial Stromal Sarcomas From Smooth Muscle Neoplasms of the Uterus. International Journal of Gynecological Pathology Official Journal of the International Society of Gynecological Pathologists, 2017. 37(4): p. 1.

20. Kurihara, S., et al., Coincident expression of $\beta$-catenin and cyclin D1 in endometrial stromal tumors and related high-grade sarcomas. Modern Pathology. 23(2): p. 225-234. 
21. De Leval, L., et al., Use of Histone Deacetylase 8 (HDAC8), a New Marker of Smooth Muscle Differentiation, in the Classification of Mesenchymal Tumors of the Uterus. American Journal of Surgical Pathology. 30(3): p. 319-327.

22. Hwang, H., et al., Immunohistochemical panel to differentiate endometrial stromal sarcoma, uterine leiomyosarcoma and leiomyoma: something old and something new. Journal of Clinical Pathology: p. jclinpath-2015-202915.

23. Franquemont, D.W., H.F. Frierson, and S.E. Mills, An Immunohistochemical Study of Normal Endometrial Stroma and Endometrial Stromal Neoplasms. American Journal of Surgical Pathology. 15(9): p. 861-870.

24. Busca, A., et al., IFITM1 Is Superior to CD10 as a Marker of Endometrial Stroma in the Evaluation of Myometrial Invasion by Endometrioid Adenocarcinoma. American Journal of Clinical Pathology. 145(4): p. 486-496.

\section{Figures}



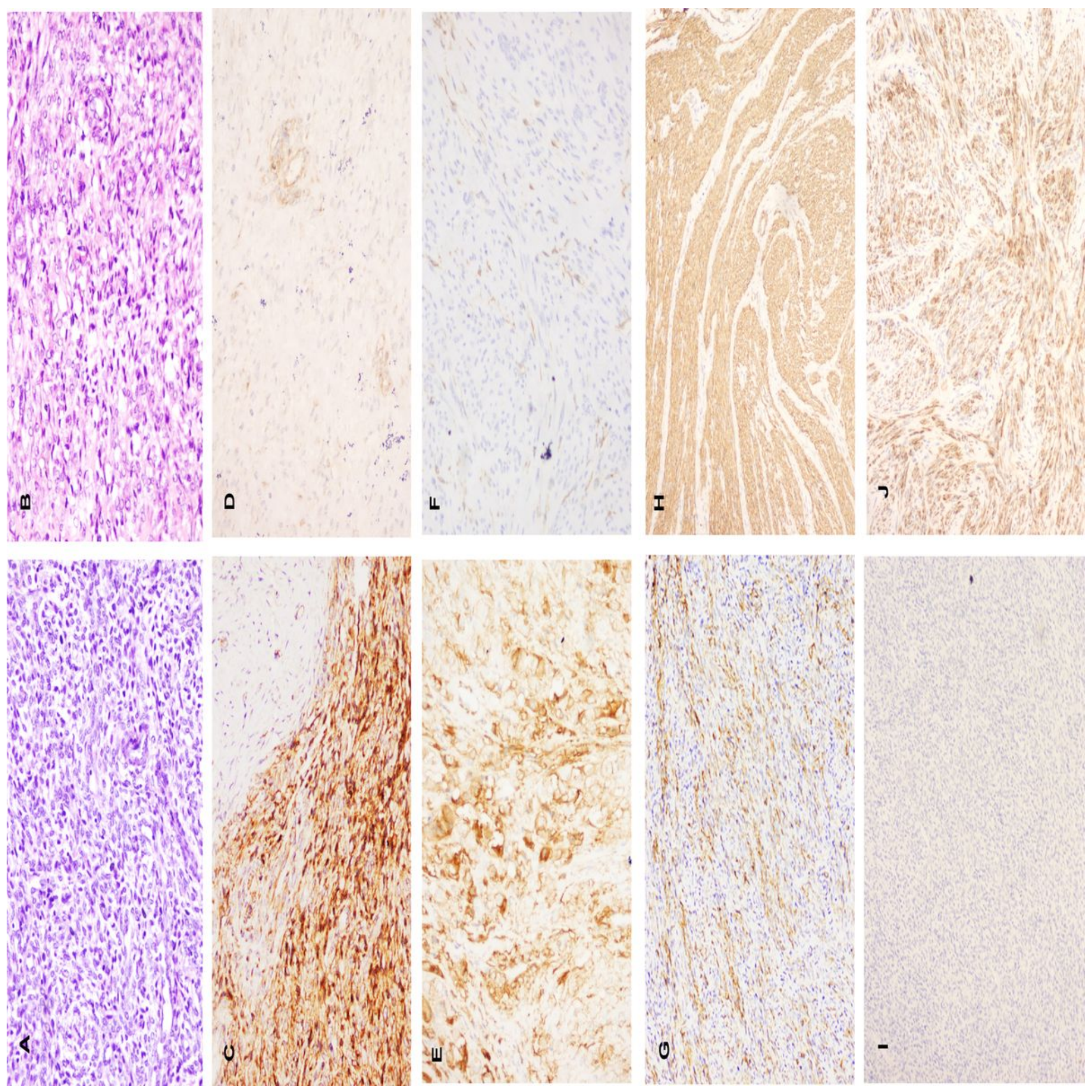

\section{Figure 1}

Immunohistochemical results. (A) Endometrial stromal sarcomas (hematoxylin and eosin stains, magnification 200). (B) Cellular leiomyomas (hematoxylin and eosin stains, magnification $\times 200$ ). (C) Endometrial stromal sarcomas showing strong positive results for IFITM1. (D) Cellular leiomyomas showing a negative or weak expression of IFITM1. (E) Endometrial stromal sarcomas exhibiting a positive expression of CD10. (F) Cellular leiomyomas exhibiting a weekly CD10 positivity. (G) Endometrial stromal sarcomas demonstrating SMA reactivity. $(\mathrm{H})$ Cellular leiomyomas showing strong positive results for 
SMA. (I) Endometrial stromal sarcomas showing a negative or weak expression of h-caldesmon. $(\mathrm{J})$ Cellular leiomyomas demonstrating strong positive results for h-caldesmon.

ROC Curve

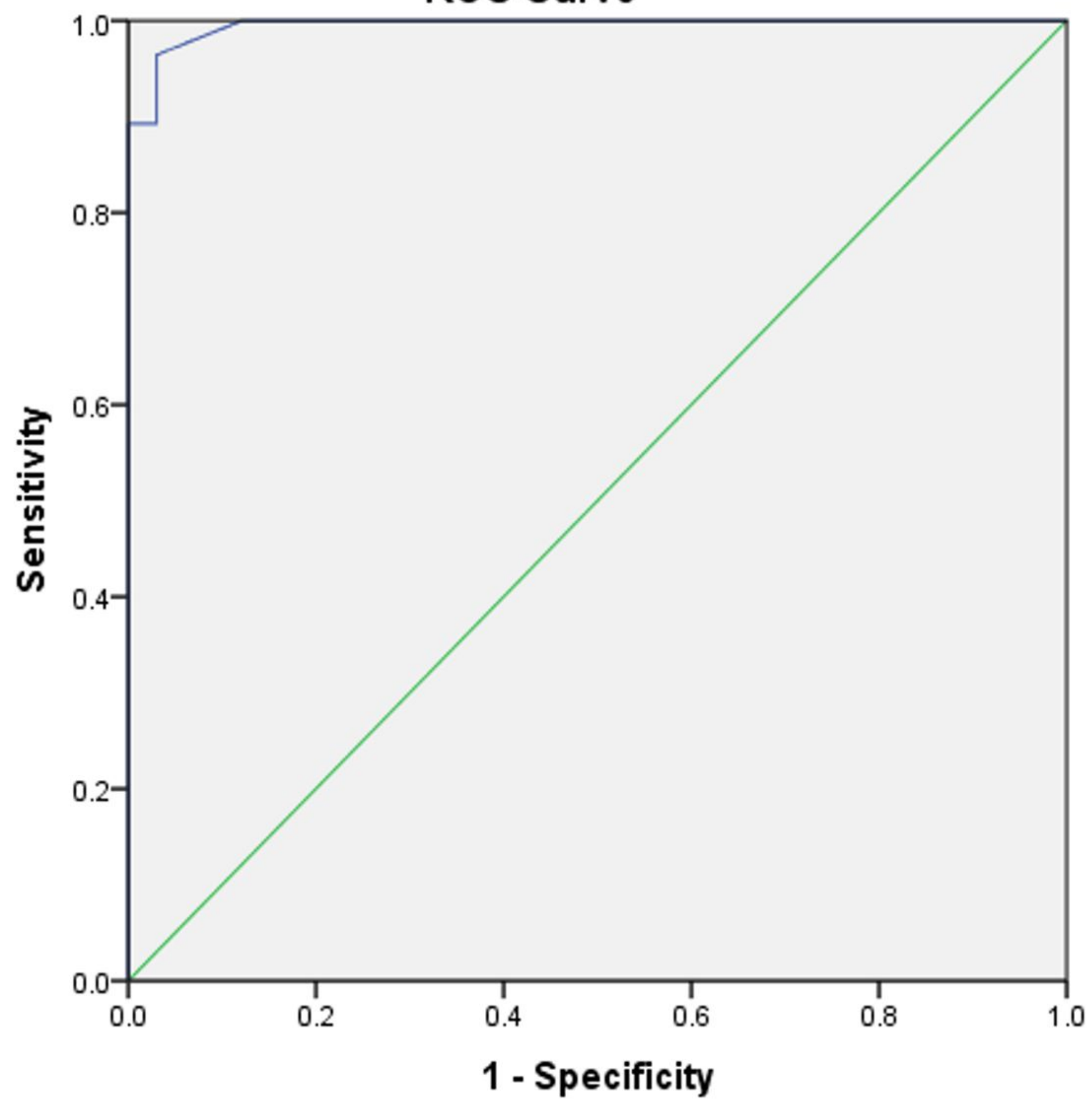

Figure 2

Receiver operating characteristic curve for prediction of Endometrial stromal sarcoma and cellular leiomyoma. 
A

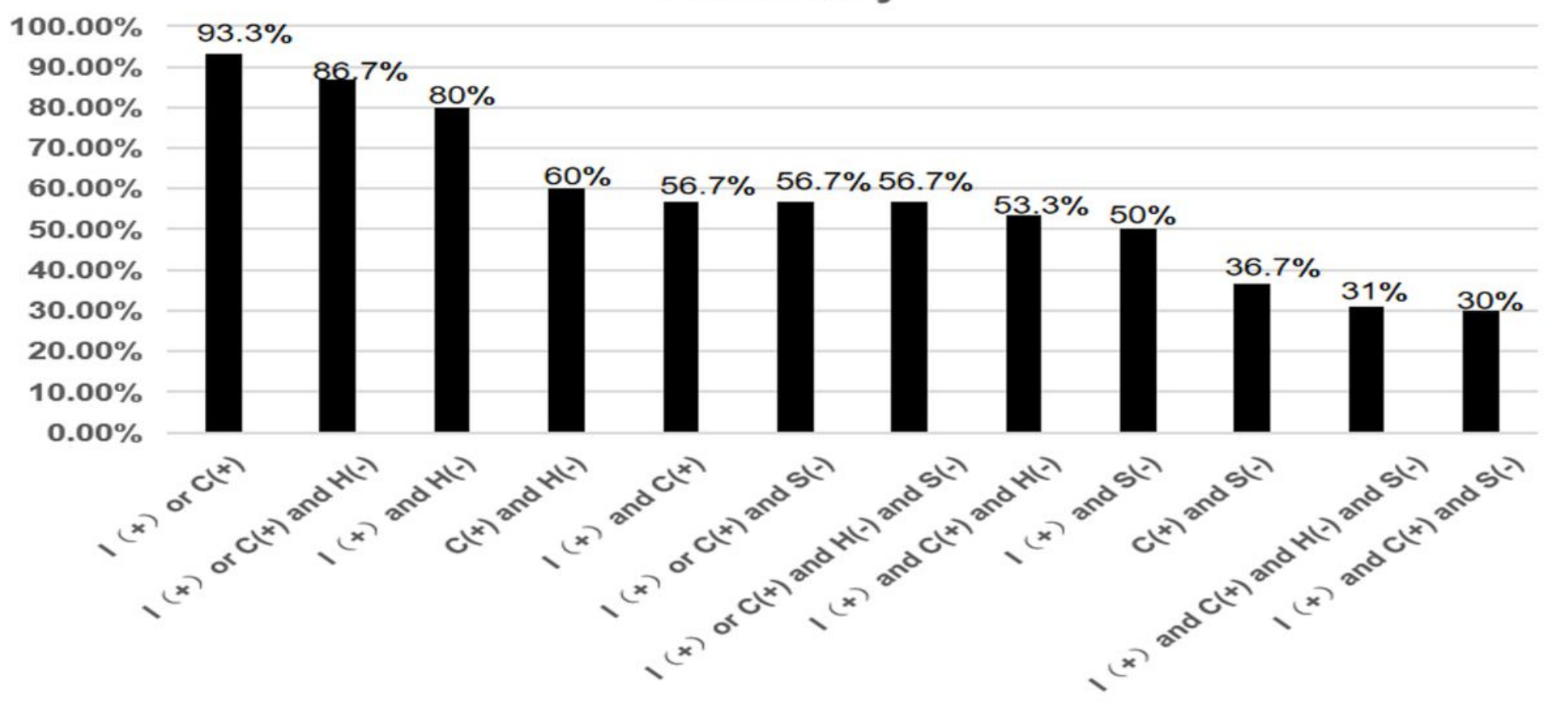

B

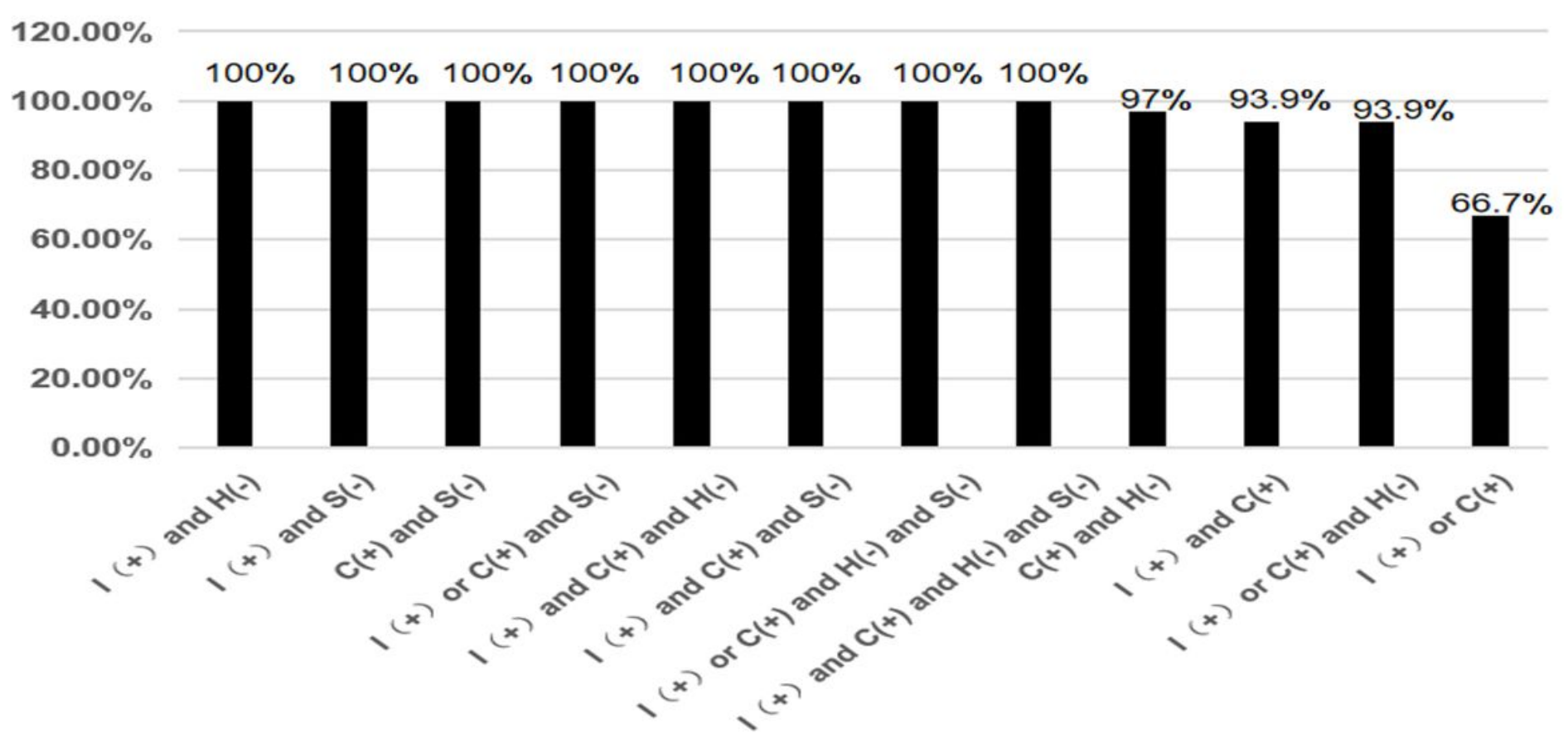

\section{Figure 3}

The Sensitivity and Specificity of combined IFITM1,CD10,h-caldesmon and SMA immunostaining in the Diagnosis of endometrial stromal sarcoma. (A) The Sensitivity of different combinations for endometrial stromal sarcoma. (B) The Specificity of different combinations for endometrial stromal sarcoma. Abbreviations: I indicates IFITM1, C indicates CD10, H indicates h-caldesmon, S indicates SMA. 


\section{Sensitivity}

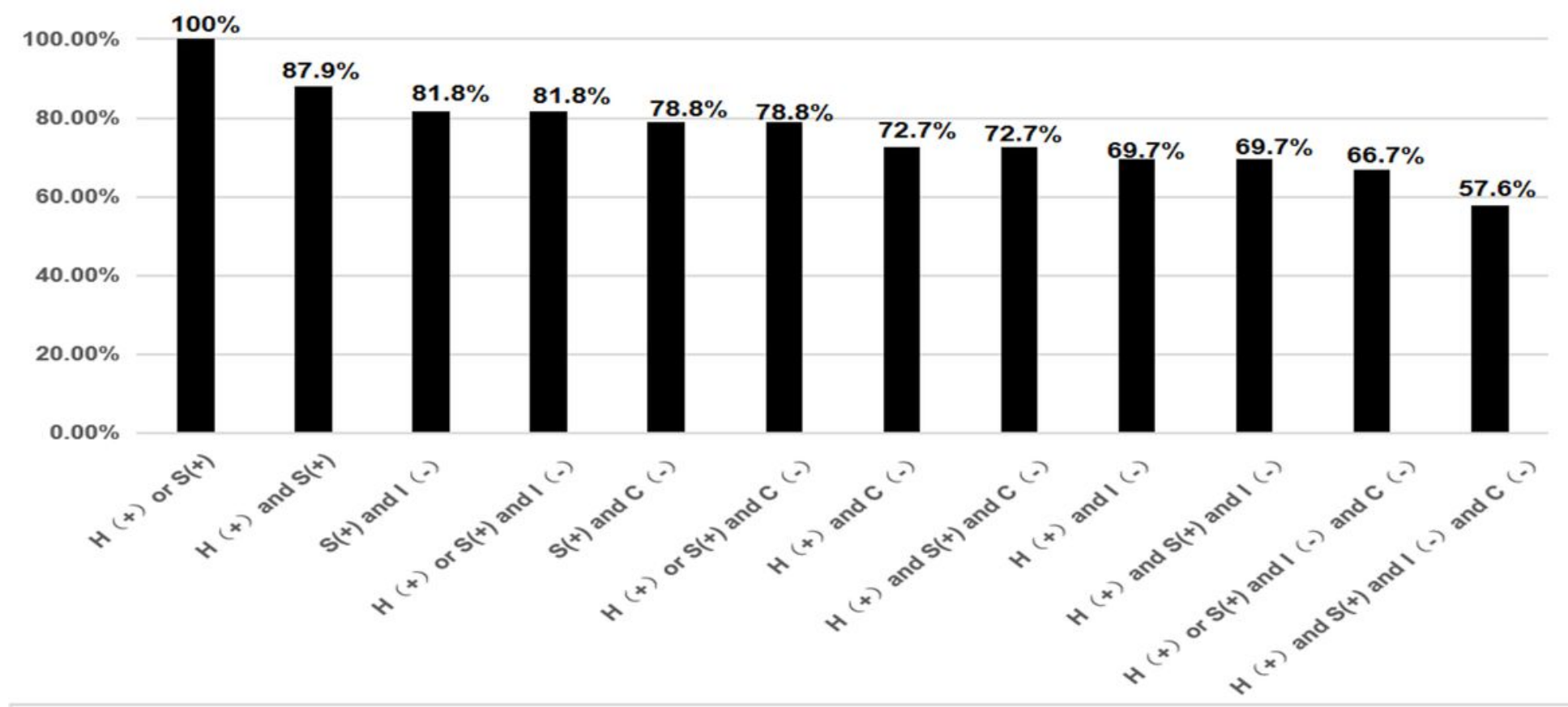

B
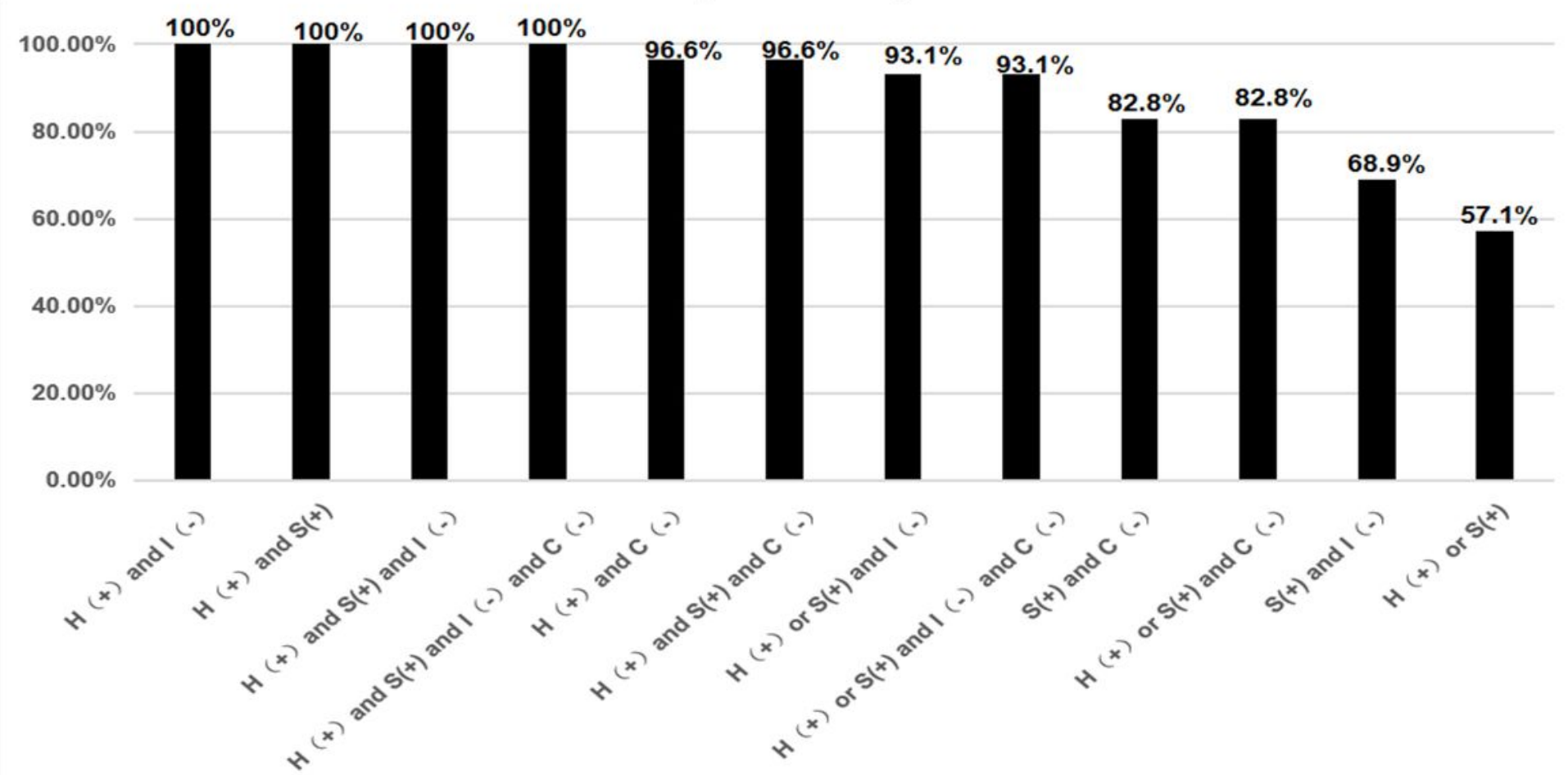

Figure 4

The Sensitivity and Specificity of combined IFITM1,CD10,h-caldesmon and SMA immunostaining in the Diagnosis of cellular leiomyoma. (A) The Sensitivity of different combinations for cellular leiomyoma. The Specificity of different combinations for cellular leiomyoma. Abbreviations: I indicates IFITM1, C indicates CD10, $\mathrm{H}$ indicates h-caldesmon, S indicates SMA. 